\title{
Hypergraph Extensions of the Erdős-Gallai Theorem
}

Ervin Győri*1, Gyula Y. Katona ${ }^{\dagger 2}$, and Nathan Lemons ${ }^{\ddagger 3}$

${ }^{1}$ Rényi Institute, Hungarian Academy of Sciences /

Department of Mathematics, Central European University, Budapest

${ }^{2}$ Department of Computer Science and Information Theory, Budapest University of Technology and Economics / MTA-ELTE Numerical Analysis and Large Networks Research Group

${ }^{3}$ Los Alamos National Labratory

December 12, 2015

\begin{abstract}
We extend the Erdős-Gallai Theorem for Berge paths in $r$-uniform hypergraphs. We also find the extremal hypergraphs avoiding $t$-tight paths of a given length and consider this extremal problem for other definitions of paths in hypergraphs.
\end{abstract}

\section{Introduction}

The aim of the present paper is to extend the following classical result to uniform hypergraphs.

*gyori.ervin@renyi.mta.hu

†kiskat@cs.bme.hu

†nlemons@lanl.gov 
Theorem 1.1 (Erdős-Gallai[3]). Let $G$ be a graph on $n$ vertices containing no path of length $k$. Then $e(G) \leq \frac{1}{2}(k-1) n$. Equality holds iff $G$ is the disjoint union of complete graphs on $k$ vertices.

The problem of finding the maximal size of a graph without a cycle of length $k$ is much harder even for ordinary graphs. However, there are some papers containing results on the hypergraph version. Since the present paper concentrates to the path version we only cite those results that are connected to the path problem.

We consider several generalizations of Theorem 1.1 for hypergraphs. This is due to the fact that there are several possible ways to define paths in hypergraphs. One such definition of paths in hypergraphs is due to Berge.

Definition 1.2. A Berge path of length $k$ in a hypergraph is a collection of $k$ hyperedges $h_{1}, \ldots, h_{k}$ and $k+1$ vertices $v_{1}, \ldots, v_{k+1}$ such that for each $1 \leq i \leq k$ we have $v_{i}, v_{i+1} \in h_{i}$.

We find the extremal sizes of $r$-uniform hypergraphs avoiding Berge paths of length $k$. Interestingly, the size of the extremal hypergraphs depend on the relationship between $r$ and $k$. Especially, the cases when $k \leq r$ and $k>r$ behave differently.

Theorem 1.3. Fix $k>r+1>3$, and let $\mathcal{H}$ be an $r$-uniform hypergraph containing no Berge path of length $k$. Then $e(\mathcal{H}) \leq \frac{n}{k}\left(\begin{array}{l}k \\ r\end{array}\right)$.

We just learned that Mubayi and Verstraete proved this theorem if $k>$ $2 r>2$ or $k>r+1>11$ but it was not published. On the other hand, if $k \leq r$, we have a different theorem. It is very annoying that the case $k=r+1$ is still open, though we think that the theorem above is right. We were able to prove it only when $r=3$ and $k=4$. The proof of this case is presented after the proof of Theorem 1.3.

Theorem 1.4. Fix $r \geq k>2$. If $\mathcal{H}$ is an $r$-uniform hypergraph with no path of length $k$, then $e(\mathcal{H}) \leq \frac{n(k-1)}{r+1}$.

Remark 1.5. Both of the above theorems are sharp for infinitely many nas the following two examples show. In the first case, if $k>r$, suppose that $k$ divides $n$ and partition the $n$ vertices into sets of size $k$. In each $k$ set, take all possible subsets of size $r$ to be in the hypergraph. Such a hypergraph has exactly $\frac{n}{k}\left(\begin{array}{l}k \\ r\end{array}\right)$ hyperedges and clearly contains no $k$-path. 
In the second case, $k \leq r$. Here we partition the vertices into sets of size $r+1$ and then on each $r+1$ set, we select exactly $k-1$ of its subsets of size $r$ to be in the hypergraph. This hypergraph has exactly $\frac{k-1}{r+1} n$ hyperedges and as each component is encompasses exactly $k-1$ edges, it is clear there is no path of length $k$. In this paper we will not deal with the case when $k=2$, as it is trivial, but it is interesting to note that the above construction is not best possible when $k=2$.

A similar result can be found in [12], the authors give upper bounds for the maximum number of edges in a hypergraph that avoids so called minimal $k$-paths. These are Berge-paths that satisfy an additional constraint that two edges of the path $h_{i}$ and $h_{j}$ are disjoint iff $|i-j| \geq 2$.

One can further restrict the idea of a Berge path, the cycle version of the following notion first appeared in [2].

Definition 1.6. Fix $r \geq 2$ and $t, 1 \leq t \leq r-1$. A $t$-tight path of length $k$ in a $r$-uniform hypergraph is a Berge-path on $k+1$ vertices $\left\{v_{1}, v_{2}, \ldots, v_{k+1}\right\}$ and $k$ hyperedges $\left\{h_{1}, h_{2}, \ldots h_{k}\right\}$ such that consecutive hyperedges intersect in at least $t$ points.

Of course a 1-tight path is the same as a Berge path. In [2] t-tight paths have been studied in other settings. A similar, but more restrictive notion called $\ell$-cycle appears in [11] yet in other context.

As in the case of Berge paths, we can get quite exact results regarding hypergraphs avoiding $t$-tight paths.

Theorem 1.7. Fix $r \geq 2$ and $t, 1 \leq t \leq r-1$. Fix $k$ large (and $n$ should be large enough too.. Let $\mathcal{H}$ be an extremal $r$-uniform hypergraph on $n$ vertices containing no t-tight path of length $k$. Then

$$
(1-o(1)) \frac{\left(\begin{array}{c}
n \\
t
\end{array}\right)\left(\begin{array}{l}
k \\
r
\end{array}\right)}{\left(\begin{array}{l}
k \\
t
\end{array}\right)} \leq e(\mathcal{H}) \leq \frac{\left(\begin{array}{c}
n \\
t
\end{array}\right)\left(\begin{array}{l}
k \\
r
\end{array}\right)}{\left(\begin{array}{l}
k \\
t
\end{array}\right)}
$$

The lower bound follows directly from a theorem of Rödl [14].

Theorem 1.8 (Rödl [14]). The packing number $m(n, k, l)$, i.e. the size of the largest $k$-uniform family of subsets of an $n$-set such that every $l$-set is contained in at most 1 member of the family is $(1+o(1)) \frac{\left(\begin{array}{l}n \\ l\end{array}\right)}{\left(\begin{array}{l}k \\ l\end{array}\right)}$. 
Remark. Just recently, Keevash improved the error term in his paper to appear.

Next we consider a further, more strict definition of a path that was first introduced by Katona and Kierstead in [10].

Definition 1.9 ([10]). A tight path of length $k$ in a $r$-uniform hypergraph is a collection of $k+r-1$ vertices $\left\{v_{1}, v_{2}, \ldots, v_{k+r-1}\right\}$ and $k$ hyperedges $\left\{h_{1}, h_{2}, \ldots h_{k}\right\}$ such that for each $1 \leq i \leq k, h_{i}=\left\{v_{i}, v_{i+1}, \ldots, v_{i+r-1}\right\}$.

It follows from the definition that a tight path is always an $(r-1)$-tight path, but not all $(r-1)$-tight paths are tight. The difference between the two definitions will be explored later on.

There are numerous papers on tight cycles, mostly in connection with Hamiltonicity. The results in $[5,15]$ are somewhat related to the present topic, bounds are given for the maximum number of edges in a uniform hypergraph with no tight Hamiltonian cycle.

For tight paths our lower and upper bounds differ by a factor $r$.

Theorem 1.10. Let $\mathcal{H}$ be an extremal $r$-uniform hypergraph containing no tight path of length $k$. Then

$$
(1-o(1)) \frac{k-r+1}{r}\left(\begin{array}{c}
n \\
r-1
\end{array}\right) \leq|e(\mathcal{H})| \leq(k-1)\left(\begin{array}{c}
n \\
r-1
\end{array}\right)
$$

The lower bound again follows easily from Rödl's Theorem 1.8.

Remark 1.11. The definition of tight paths in uniform hypergraphs can be extended to tight trees in $r$-uniform hypergraphs. Such trees are defined inductively. A single edge forms a tight 1 tree. If a collection of edges form a tight $(k-1)$ tree, then adding a new edge which intersects a previous edge in $r-1$ vertices and contains a 'new' vertex (which is not contained in any of the previous edges) yields a tight $k$ tree. It is easy to see that tight paths are also tight trees. Kallai [4] made the following conjecture regarding the extremal number for tight trees.

Conjecture 1.12 (Kalai [4]). If the number of edges in an r-uniform hypergraph is $>\frac{k-1}{r}\left(\begin{array}{c}n \\ r-1\end{array}\right)$ then it contains every $r$-tree with $k$ edges.

In [4] the authors prove a special case of this conjecture.

Note that if true, the conjecture would imply that the upper bound in Theorem 1.10 is $\frac{k-1}{r}\left(\begin{array}{c}n \\ r-1\end{array}\right)$. 
As noted above, there is an important difference between $(r-1)$-tight paths and tight paths in $r$-uniform hypergraphs. We can investigate this difference by considering the following.

Definition 1.13. Let $k>r \geq 2$ and $1 \leq J<k$. Then a Berge path of length $k$ in a $r$-uniform hypergraph $\mathcal{H}$ on hyperedges $e_{1}, \ldots, e_{k}$ satisifies intersection conditions $(J)$ if

$$
\text { for } 1 \leq l \leq J \text { and } i>l, \quad\left|e_{i} \cap e_{i-l}\right|=\max \{r-l, 0\}
$$

Of course a Berge path satisfying intersection conditions (1) is the same as an $r-1$ tight path. Furthermore, a Berge path satisfying intersection conditions $(k-1)$ is exactly a tight path. It is interesting that as above, an extremal hypergraph excluding $(r-1)$-tight paths of length $k$ contains assymptotically $\frac{k-r+1}{r}\left(\begin{array}{c}n \\ r-1\end{array}\right)$ hyperedges. On the other hand, our best construction for a hypergraph exluding a tight path of lenght $k$ contains $\frac{k-1}{r}\left(\begin{array}{c}n \\ r-1\end{array}\right)$ hyperedges. Supposedly, each Berge path satisfying intersection conditions (J) falls somewhere between these two. However, while there are $k-1$ different intersection conditions, there are only $r-1$ possible block sizes in Theorem 1.8 (which we believe form the extremal hypergraphs).

The rest of the paper is organized as follows. In section 2 we prove Theorem 1.3. No really new new ideas are needed; our proof is very similar to the original one. In section 3 we look at $t$-tight paths. In section 4 we consider tight paths and $(r-1)$-tight paths satisfying a certain number of intersection conditions as in Definition 1.13.

Remark. An extended abstract of our results already appeared in [9]. Since that several papers cited them in various context: $[6,8,13]$, furthermore Allen, Böttcher, Cooley and Mycroft [1] have obtained upper and lower bounds for an analogue of Theorem 1.10 in the range $k=\alpha n$ for $\alpha$ constant, which are asymptotically almost equal when $\alpha$ is small.

\section{Berge Paths}

Proof of Theorem 1.3. Let $P$ be a longest path in $\mathcal{H}$. Let $v_{1}, v_{2}, \ldots, v_{l+1}$ be the vertices of $P$, and $h_{1}, h_{2}, \ldots, h_{l}$ the hyperedges such that for each $i=1, \ldots l, v_{i}, v_{i+1} \in h_{i}$. Let $\mathcal{H}^{\prime}$ be the hypergraph obtained by deleting the edges of $P$ from $\mathcal{H}$. Specifically, let $\mathcal{H}^{\prime}=\mathcal{H} \backslash\left\{h_{1}, h_{2}, \ldots, h_{l}\right\}$. Suppose that $l<k$. 
Lemma 2.1. If there is a cycle of length $l+1$ on the vertices $v_{1}, v_{2}, \ldots, v_{l+1}$, then these vertices constitute a component of the hypergraph $\mathcal{H}$.

Proof. To see this, suppose that $C$ is such a cycle. Then if an edge $h$ in the cycle $C$ does not lie completely within the vertices $v_{1}, v_{2}, \ldots, v_{l+1}$, then deleting $h$ from $C$ we have an $l$-path which can be extended (by the edge $h$ ) to a path of length at least $l+1$. Thus every edge $h$ in the cycle $C$ must be contained within the vertices $v_{1}, v_{2}, \ldots, v_{l+1}$. In fact, something stronger is true. For each vertex in the cycle, $v_{i}$, the neighborhood of $v_{i}$ lies within $v_{1}, v_{2}, \ldots, v_{l+1}$. (The neighborhood of a vertex is the set of vertices in $\mathcal{H}$ which are connected to $v_{i}$ by an edge.) Indeed, suppose that for some $i$, the vertex $v_{i}$ has a neighbor $y$ outside of $\left\{v_{1}, v_{2}, \ldots, v_{l+1}\right\}$. Then the edge containing both $v_{i}$ and $y$ is not an edge of $C$ (by the above argument.) Thus, removing an appropriate edge of $C$ so that it is a path of length $l$ with $v_{i}$ as an endvertex, we can extend this to a path of length $l+1$ with $y$ as an endvertex, a contradiction.

Based on Lemma 2.1, we prove the theorem by induction on $n$. Clearly, for small values of $n$, the theorem trivially holds. Now, fix $n$ such that the theorem holds for all $n^{\prime}<n$ Then let $\mathcal{H}=(\mathcal{E}, V)$ be a $r$-uniform hypergraph on $n$ vertices with $e(\mathcal{H})>\frac{n}{k}\left(\begin{array}{l}k \\ r\end{array}\right)$. We can asssume that the following holds for the minimal degree, $\delta$, in $\mathcal{H}$.

$$
\delta=e(\mathcal{H})>\frac{1}{r}\left(\begin{array}{l}
k-1 \\
r-1
\end{array}\right)
$$

Otherwise, if there is a vertex $x$ in $\mathcal{H}$ with degree no more than $\left(\begin{array}{l}k-1 \\ r-1\end{array}\right)$, then we may delete this vertex (and all the edges incident with it) from $\mathcal{H}$. The result will be a hypergraph on $n-1$ vertices with more than $\frac{n-1}{k}\left(\begin{array}{l}k \\ r\end{array}\right)$ edges. Thus by the induction hypothesis, this hypergraph will have a path of length $k$.

Let $P$ be a longest path in $\mathcal{H}$. Let $v_{1}, v_{2}, \ldots, v_{l+1}$ be the vertices of $P$, and $h_{1}, h_{2}, \ldots, h_{l}$ the hyperedges such that for each $i=1, \ldots l, v_{i}, v_{i+1} \in h_{i}$. Suppose that $l<k$.

Let $\mathcal{H}^{\prime}$ be the hypergraph obtained by deleting the edges of $P$ from $\mathcal{H}$. Specifically, let $\mathcal{H}^{\prime}=\mathcal{H} \backslash\left\{h_{1}, h_{2}, \ldots, h_{l}\right\}$. Note that by the choice of $P$, the neighborhoods of $v_{1}$ and $v_{l+1}$ in $\mathcal{H}^{\prime}$ must fall within $\left\{v_{1}, v_{2}, \ldots, v_{l+1}\right\}$

Claim 2.2. We may assume that $v_{l+1}$ (and similarly $v_{1}$ ) is contained by at least one hyperedge in $\mathcal{H}^{\prime}$. 
Proof of Claim 2.2. Suppose $v_{l+1}$ is not contained by any hyperedge in $\mathcal{H}$. Let $S$ be a maximal set of vertices $v_{i}$ of $P$ such that the vertices and edges of $P$ can be reordered to constitute a path $P^{\prime}=v_{1}^{\prime}, h_{1}^{\prime}, v_{2}^{\prime}, \ldots, v_{l}^{\prime}, h_{l}^{\prime}, v_{l+1}^{\prime}$ such that

(1) $v_{1}^{\prime}=v_{1}$

(2) $v_{l+1}^{\prime}=v_{i}$

(3) if $v_{j-1}, v_{j} \notin S$ then $v_{j-1}$ and $v_{j}$ are consecutive (there are two possible orders!) and they are joined by the hyperedge $h_{j-1}$.

If $v$ is contained in a hyperedge not in $P$ then we are done.

Notice that $\left\{v_{l+1}\right\}$ is such a set, so $S$ is nonempty. Suppose that $h_{j}$ is a hyperedge such that $v_{j-1}, v_{j} \notin S$ but $h_{j}$ contains a vertex $v \in S$. Then take the path $P^{\prime}$ satisfying (1)-(3) with $v_{l+1}^{\prime}=v$. Then take the starting segment of $P^{\prime}$ to the first vertex of $v_{j-1}$ and $v_{j}$, then continue with $h_{j}$ and the segment of $P^{\prime}$ backward from $v$ to the second vertex of $v_{j-1}$ and $v_{j}$. By definition, the last vertex of this path should belong to $S$, a contradiction. So if we delete a vertex $v_{j} \in S$ from $V(\mathcal{H})$ then we delete just the hyperedges $h_{j-1}, h_{j}$ from $\mathcal{H}$, so at most $2|S|-1$ edges and the proof of the Claim is complete by the induction hypothesis, since $\frac{1}{r}\left(\begin{array}{l}k-1 \\ r-1\end{array}\right) \geq 2$.

Clearly, if there is an edge of $\mathcal{H}^{\prime}$ containing both $v_{1}$ and $v_{l+1}$, then the edges of $P$ form a cycle of length $l+1$ and we are done as above. On the other hand, if there exist edges $g_{1}, g_{2} \in \mathcal{H}^{\prime}$ such that for some $i, 1<i<l+1$, $v_{1}, v_{i+1} \in g_{1}$ and $v_{l}+1, v_{i} \in g_{2}$ then clearly $k \geq 2 r$ and there is an $(l+1)$-cycle again on the vertices

$$
v_{1}, v_{i+1}, v_{i+2}, v_{i+3}, \ldots, v_{l+1}, v_{i}, v_{i-1}, v_{i-2}, \ldots, v_{1} .
$$

Thus by the pigeonhole principle, if in $\mathcal{H}^{\prime}$ the degrees of both $v_{1}$ and $v_{l+1}$ are greater than $\left(\frac{\frac{k-2}{2}}{r-1}\right)$, then there is a $l+1$ cycle on $v_{1}, v_{2}, \ldots, v_{l+1}$ in $\mathcal{H}$. If the degree of both $v_{1}$ and $v_{l+1}$ are at most $\left(\begin{array}{c}\frac{k-2}{2} \\ r-1\end{array}\right)$, then delete these vertices and the hyperedges in $P$ and we are done since

$$
\frac{2}{r}\left(\begin{array}{l}
k-1 \\
r-1
\end{array}\right) \geq 2\left(\begin{array}{c}
\frac{k-2}{2} \\
r-1
\end{array}\right)+k-1
$$

if $k \geq 2 r$. (We leave the details to the reader.)

Finally if, say, the degree of $v_{1}$ is greater than $\left(\frac{k-2}{r-1}\right)$ but the degree of $v_{l+1}$ is at most $\left(\begin{array}{c}\frac{k-2}{2} \\ r-1\end{array}\right)$ then the degree of $v_{l+1}$ is at most $\left(\begin{array}{c}\frac{k-2}{2} \\ r-1\end{array}\right)+\frac{k-2}{2}$ since if $v_{j}$ is 
contained by a hyperedge $e$ in $\mathcal{H}^{\prime}$ and $h_{j-1}$ contains $v_{l+1}$ then $e, h_{1}, \ldots h_{j}-$ $1, h_{l}, h_{l-1}, \ldots h_{j}$ constitute a cycle and we are done by induction. So the degree of $v_{l+1}$ is at most

$$
\frac{1}{r}\left(\begin{array}{l}
k-1 \\
r-1
\end{array}\right) \geq\left(\begin{array}{c}
\frac{k-2}{2} \\
r-1
\end{array}\right)+\frac{k-1}{2}
$$

and we are done again by induction as above if we delete the vertex $v_{l+1}$ and the hyperedges containing it.

This completes the proof of the theorem.

Conjecture 2.3. Fix $k=r+1>2$, and let $\mathcal{H}$ be an $r$-uniform hypergraph containing no Berge path of length $k$. Then $e(\mathcal{H}) \leq \frac{n}{k}\left(\begin{array}{l}k \\ r\end{array}\right)=n$.

We can prove the conjecture if $r=3$ and $k=4$.

Theorem 2.4. Let $\mathcal{H}$ be a 3-uniform hypergraph containing no Berge path of length 4 . Then $e(\mathcal{H}) \leq n$.

Proof. The following claim is an easy exercise to prove.

Claim 2.5. If a connected graph on $n$ vertices contains at least $n+1$ edges and does not contain a path of length 4 (with 5 different vertices) then it is either $a K_{4}$ or $K_{4}-e$.

To prove the theorem we use the method introduced in [7]. Suppose indirectly that there exists a 3 -uniform hypergraph $\mathcal{H}$ without a path of length 4 that contains at least $n+1$ edges. If the hypergraph is not connected then at least one of the components must have more edges than vertices, so we can assume that our hypergraph is connected.

Let us construct a graph $H$ on the ground set of $\mathcal{H}$ by embedding a (unique) edge into each hyperedge of $\mathcal{H}$. Construct $H$ greedily, take the hyperedges of $\mathcal{H}$ in arbitrary order and for each hyperedge embed an edge that has not already been used in $H$.

If at some step we cannot find such an edge, then $\mathcal{H}$ contains the edge $e_{0}=\left\{v_{1}, v_{2}, v_{3}\right\}$ so that each edge in $H$ is already assigned to a hyperedge. Let these edges be $e_{1}=\left\{v_{1}, v_{2}, u_{1}\right\}, e_{2}=\left\{v_{2}, v_{3}, u_{2}\right\}, e_{3}=\left\{v_{3}, v_{1}, u_{3}\right\}$. If $u_{1}=$ $u_{2}=u_{3}$ then $u_{1}, e_{1}, v_{1}, e_{0}, v_{2}, e_{2}, v_{3}, e_{3}, u_{1}$ is a cycle of length 4 , so by Lemma 2.1 these 4 vertices and 4 edges form a component, contradicting the assumption. On the other hand, if say $u_{1} \neq u_{2}$, then $u_{1}, e_{1}, v_{1}, e_{0}, v_{2}, e_{2}, v_{3}, e_{3}, u_{2}$ is a path of length 4 . 
Thus we can assume that the greedy algorithm assigned an edge to each hyperedge. If $H$ contains a path of length 4 on 5 different vertices then a hyperedges that were assigned to the edges to the path clearly form a path of length 4 in $\mathcal{H}$, a contradiction again. Thus by Claim 2.5 every component of $H$ is a $K_{4}$ or $K_{4}-e$. Take one such component. It clearly contains a cycle of length 4 , therefore the corresponding hyperedges form a cycle of length 4 . Using Lemma 2.1 we get a contradiction again.

We now consider the case of $r$-uniform hypergraphs avoiding a Berge path of length $k$ where $r \geq k>2$.

Proof of Theorem 1.4. We will prove Theorem 1.4 by induction on $k$. In fact, we prove a bit stronger statement.

Proposition 2.6. Fix $k$ and $r$ such that $r \geq k>2$. Let $H$ be a connected $r$-uniform hypergraph with

$$
e(H)>\frac{k-1}{r+1} n
$$

where $n$ is the number of vertices in $H$. Then for each edge $e \in H$, there is a Berge path of length $k$ in $H$ starting with $e$.

It is easy to see that the proposition is a strengthening of Theorem 1.4.

Proof of Proposition 2.6. By induction on $k$.

We first consider the case $k=3$. Suppose the theorem does not hold and let $H$ be a minimal (in terms $n$ ) counterexample. Then by assumption, there exists an edge $e \in H$ such that all paths starting with $e$ in $H$ are of length $k-1$ or less. We will show this leads to a contradiction.

If there exists an edge $f \in H$ disjoint from $e$ then, as $H$ is connected, there must be a 3-path starting at $e$. We can thus suppose that every edge of $H$ meets $e$. Suppose that two edges $f$ and $g$ of $H$ meet outside $e$. Clearly $e, f, g$ form a 3-path. On the otherhand if there exist edges $f$ and $g$ such that $|e \cap f| \geq 2$ and $|e \cap f \cap g| \geq 1$ then again $e, f, g$ form a 3-path. Thus we may assume that every edge of $H$ meets $e$, no edges meet outside of $e$ and an edge meeting $e$ in at least 2 vertices meets no other edges of $H$.

We can now count the $n$ vertices of $H$. First there are the $r$ vertices in $e$. Then there is at most one edge, $f$, which intersects $e$ in two or more points; this edge contributes at least one new vertex to the count. The remaining 
edges each intersect $e$ in one point and thus each contribute $r-1$ vertices to the count: $n \geq(r-1)(e(\mathcal{H})-1)+2$. But then as $n \geq r+1$,

$$
\begin{aligned}
e(H) & \leq \frac{n-2}{r-1}+1 \\
& \leq \frac{2}{r+1} n,
\end{aligned}
$$

contradicting the inequality in Proposition 2.6.

Now suppose the theorem holds for $k-1$, where $k \geq 4$ is fixed. Let $H$ a connected $r$-uniform hypergraph satisfying the inequality in Proposition 2.6. Fix an edge $e$ in $H$. The basic idea is to remove $e$ from $H$ and apply induction on the remaining graph to find a $k-1$ path $P$, such that $P+e$ forms a $k$ path. The only difficulty arise in finding an apropriate subgraph in which to apply the inductive hypothesis. To this end, consider the components of $H-e: C_{1}, C_{2}, \ldots, C_{m}$. We claim there must be an $i$ such that

$$
e\left(C_{i}\right)+1>\frac{k-1}{r+1} v\left(C_{i}\right)
$$

Otherwise we get the contradiction

$$
e(H) \leq \sum e\left(C_{i}\right)+1 \leq \sum \frac{k-1}{r+1} v\left(C_{i}\right) \leq \frac{k-1}{r+1} n .
$$

Now pick a vertex $x \in e \cap C_{i}$ and let $C_{i}-x$ be the (possibly no longer uniform) hypergraph obtained by removing $x$ from every edge of $C_{i}$ : $C_{i}-x=\{g-x \mid g \in$ $C_{i}$ \}. Let $C_{i 1}, C_{i 2}, \ldots, C_{i t}$ be the connected componenets of $C_{i}-x$. It can be checked that for $r \geq k, e\left(C_{i}\right)+1>\frac{k-1}{r+1} v\left(C_{i}\right)$ implies $e\left(C_{i}\right)>\frac{k-2}{r}\left(v\left(C_{i}\right)-1\right)$. Thus there exists a $j$ such that

$$
e\left(C_{i j}\right)>\frac{k-2}{r} v\left(C_{i j}\right)
$$

Let $e^{*}$ be an edge of $C_{i j}$ for which the edge $e^{*} \cup\{x\}$ belongs to $C_{i}$. To complete the proof, we will reduce the $r$-edges of $C_{i j}$ each by one vertex to achieve a $(r-1)$-uniform hypergraph, $H^{*}$, connected and satisfying

$$
e\left(H^{*}\right)>\frac{k-2}{r} v\left(H^{*}\right) .
$$

We will then use induction to find a $k-1$ path starting at $e^{*}$ in $H^{*}$. To be able to apply the inductive hypothesis, we must ensure that the process 
of reducing the $r$ edges of $C_{i j}$ to $r-1$ edges neither disconnects the graph nor creates multiple edges. We claim that, one by one, for each $r$-edge of $C_{i j}$, we can pick a vertex of the edge and remove it from the edge such that the remaining graph is still connected and such that no multiple edges are created.

Suppose for some $r$-edge $f$ this is not possible. If every vertex of $f$ is a cut vertex, then no other edge meets $f$ in more than one vertex and we simply contract the vertices of $f$ to one vertex and delete $f$ from the graph. The graph is still connected, there are no multiple edges created in this step (otherwise not every vertex of $f$ would be a cut-vertex) and it can be checked that

$$
\frac{(k-2)(r-1)}{r} \geq 1
$$

which holds for $r \geq k \geq 4$, implies that

$$
e\left(C_{i j}\right)-1>\frac{k-2}{r}\left(v\left(C_{i j}\right)-(r-1)\right) .
$$

Thus we may assume that not every vertex of $f$ is a cut vertex. Suppose now that the deletion of any vertex of $f$ would lead to multiple edges in the graph. This means that every $r-1$ subset of $f$ is already an edge of the graph. In this case there is clearly a $P_{r-1}$ path within the edge $f$. This path can be extended (in the original graph $H$ ) to the edge $e$; such a path will have length at least $k$.

Finally it is clear that if removing a vertex $x$ from $f$ causes a multiple edge to appear in the graph then there cannot be another vertex $y$ of $f$ whose removal (from $f$ ) would cut the graph. Thus we can indeed transform $C_{i j}$ into a $(r-1)$ uniform, connected hypergraph satisfying

$$
e\left(H^{*}\right)>\frac{k-2}{r} v\left(H^{*}\right)
$$

In particular, by the induction hypothesis, there is a $k-1$ path in $H^{*}$ starting at $e^{*}$. Let $e^{*}, e_{2}^{*}, \ldots, e_{k-1}^{*}$ be the edges of this path and consider the associated edges in $H: e_{1}, e_{2}, \ldots, e_{k-1}$. Now by definition of $H^{*}, x \in e \cap e_{1}$ and $x \notin$ $\cup_{l>2} e_{l}^{*}$. Thus we can extend the path in $H$ to $e, e_{1}, e_{2}, \ldots, e_{k-1}$ except in the case when $r=k$ and $e$ consists precisely in those vertices in the intersections $e_{l} \cap e_{l+1}$ together with the endpoint of the path in $e_{k-1}$. However as $e_{k-1}$ must be different from $e$, we may simply choose a different endpoint in $e_{k-1}$. 


\section{3 t-Tight Paths}

Proof of Theorem 1.7. First consider the lower bound. By Theorem 1.8, there is a family $\mathcal{B}$ of $k$-sets of an initial $n$ set such that no $t$ set is contained in more than 1 element of the family and such that

$$
|\mathcal{B}| \geq(1-o(1)) \frac{\left(\begin{array}{l}
n \\
t
\end{array}\right)}{\left(\begin{array}{l}
k \\
t
\end{array}\right)}
$$

We claim that the $r$-uniform hypergraph $\mathcal{H}$ obtained by replacing each member of $\mathcal{B}$ with all its $\left(\begin{array}{l}k \\ r\end{array}\right) r$-sets contains no $t$-tight path. Any such path would have vertices in at least 2 different memebers of $\mathcal{B}$. Specifically, such a path would contain vertices $u$ and $v$ with $u \in B_{1} \backslash B_{2}$ and $v \in B_{2} \backslash B_{1}$ where $B_{1}$ and $B_{2}$ are two distinct members of $\mathcal{B}$. But $\left|B_{1} \cap B_{2}\right|<t$ (the same holds for all distinct pairs of members of $\mathcal{B}$.) Thus there can be no $t$-tight path in $\mathcal{H}$ from $u$ to $v$.

We now look at the upper bound. If $t=1$ then we are done by Theorem 1.3. Suppose then that $t \geq 2$. Let $\mathcal{H}$ be a hypergraph on $n$ vertices with more than $\left(\begin{array}{l}n \\ t\end{array}\right)\left(\begin{array}{l}k \\ r\end{array}\right) /\left(\begin{array}{l}k \\ t\end{array}\right)$ hyperedges. Then it is easy to see that there exists a vertex $x_{1} \in V$ with degree at least

$$
\frac{r}{n} \frac{\left(\begin{array}{c}
n \\
t
\end{array}\right)\left(\begin{array}{l}
k \\
r
\end{array}\right)}{\left(\begin{array}{l}
k \\
t
\end{array}\right)}=\frac{\left(\begin{array}{c}
n-1 \\
t-1
\end{array}\right)\left(\begin{array}{l}
k-1 \\
r-1
\end{array}\right)}{\left(\begin{array}{c}
k-1 \\
t-1
\end{array}\right)}
$$

Let $\mathcal{H}_{1}=\left\{h \backslash\left\{x_{1}\right\}: h \in \mathcal{H} \wedge x_{1} \in h\right\}$ be the link of $x_{1}$. Then continuing we can clearly find vertices $x_{2}, \ldots, x_{t-1}$ such that for $1<i<t, \mathcal{H}_{i}$ is the link of $x_{i}$ in $\mathcal{H}_{i-1}$ and such that for $1<i<t$, the degree of $x_{i}$ in $\mathcal{H}_{i-1}$ is greater than $\left(\begin{array}{c}n-i \\ t-i\end{array}\right)\left(\begin{array}{c}k-i \\ r-i\end{array}\right) /\left(\begin{array}{c}k-i \\ t-i\end{array}\right)$. But then $\mathcal{H}_{t-1}$ is simply a $(r-t+1)$-graph on $n-t+1$ vertices with more than $\frac{n-t+1}{k-t+1}\left(\begin{array}{c}k-t+1 \\ r-t+1\end{array}\right)$ edges. But then applying Theorem 1.3 we can find a path of length $k-t+1$ in $\mathcal{H}_{t-1}$. If the minimal degree in $\mathcal{H}_{t-1}$ is large enough, we can then extend this path using the vertices $x_{1}, \ldots, x_{t-1}$ to a $t$-tight path of length $k$ in $\mathcal{H}$.

\section{Even Tighter Paths}

In this section we consider the relationship between tight and $t$-tight paths. We prove Theorem 1.10 and a related theorem concerning $(r-1)$-tight paths satisfying intersection conditions $J$ for fixed $1 \leq J \leq k-1$. First we will need a simple averaging argument. 
Lemma 4.1. Let $\mathcal{H}$ be an $r$-uniform hypergraph on $n$ vertices with strictly more than $c\left(\begin{array}{c}n \\ r-1\end{array}\right)$ edges. Then there exists an nonempty sub-hypergraph, $\mathcal{H}^{\prime}$, of $\mathcal{H}$ such that

$$
\forall S \in\left(\begin{array}{c}
\mathcal{V}(\mathcal{H}) \\
r-1
\end{array}\right), d_{\mathcal{H}^{\prime}}(S) \leq c \Rightarrow d_{\mathcal{H}^{\prime}}(S)=0
$$

where $d_{\mathcal{H}^{\prime}}(S)$ refers to the number of hyperedges of $\mathcal{H}^{\prime}$ containing the set $S$.

Proof of Lemma 4.1. Let $\mathcal{H}$ be as in the statement of the lemma, and let $\mathcal{H}_{1}$ be the $(r-1)$-uniform hypergraph on $\mathcal{V}(\mathcal{H})$ with edge set $\left\{e \in\left(\begin{array}{c}\mathcal{V}(\mathcal{H}) \\ r-1\end{array}\right): \exists h \in\right.$ $\mathcal{H}, e \subset h\}$. The hypergraph $\mathcal{H}_{1}$ is commonly called the lower shadow of $\mathcal{H}$. Let $w$ be a weight function on the edges of $\mathcal{H}_{1}$ where $w(e)=\mathrm{d}_{\mathcal{H}}(e)$. Then

$$
\sum_{e \in \mathcal{H}_{1}} w(e)=r \cdot \mathrm{e}(\mathcal{H})>r c\left(\begin{array}{c}
n \\
r-1
\end{array}\right)
$$

and the average weight, $\bar{w}$, over the edges of $\mathcal{H}_{1}$ is strictly more than $r c\left(\begin{array}{c}n \\ r-1\end{array}\right)$. Let $e$ be an edge of $\mathcal{H}_{1}$ with weight no more than $c$. Now let $\mathcal{H}^{\prime}=\mathcal{H} \backslash\{h: e \subset$ $h$ \} be a subgraph of $\mathcal{H}$ and let define a new weight function $w^{\prime}$ on the edges of $\mathcal{H}_{1}: w^{\prime}(g)=\mathrm{d}_{\mathcal{H}^{\prime}}(g)$ for each $g \in \mathcal{H}_{1}$. Finally let $\mathcal{H}_{1}^{\prime}=\mathcal{H}_{1} \backslash\left\{g: w^{\prime}(g)=0\right\}$. Then

$$
\sum_{g \in \mathcal{H}_{1}^{\prime}} w^{\prime}(g)=\sum_{g \in \mathcal{H}_{1}} w^{\prime}(g) \geq \sum_{g \in \mathcal{H}_{1}} w(g)-r c>r c \cdot \mathrm{e}\left(\mathcal{H}_{1}^{\prime}\right)
$$

and in particular, $\left(1 / \mathrm{e}\left(\mathcal{H}_{1}^{\prime}\right)\right) \sum_{g \in \mathcal{H}_{1}^{\prime}} w^{\prime}(g)>r c$. Replacing $\mathcal{H}$ with $\mathcal{H}^{\prime}$ and $\mathcal{H}_{1}$ with $\mathcal{H}_{1}^{\prime}$, we may repeat the above operation (as long as there are edges $e \in \mathcal{H}_{1}$ with degree no more than $c$ and the average weight of the resulting hypergraph $\mathcal{H}_{1}^{\prime}$ will always be bounded below by $r c$. In particular at some point there will be no more edges of degree less than or equal to $c$ in $\mathcal{H}_{1}^{\prime}$, and at that point $\mathcal{H}^{\prime}$ will have the desired property. Note that $\mathcal{H}^{\prime}$ will not be empty as the average degree of $(r-1)$ sets in the shadow of $\mathcal{H}^{\prime}$ will be bounded below by $r c$.

Proof of Theorem 1.10. The lower bound follows from our usual construction using Theorem 1.8. The upper bound follows just as easily from Lemma 4.1. If an $r$-uniform hypergraph $\mathcal{H}$ on $n$ vertices satisfies 2 then it is quite clear that we can find a tight path of length $k$ in $\mathcal{H}$ : in fact every edge of $\mathcal{H}$ will be contained in such a path. 
It is thus perhaps unsurprising that the upper bound (and trivially the lower bound) of Theorem 1.7 for $(r-1)$-tight paths also holds for paths satisfying intersection conditions (2) if $k$ is big enough compared to $r$. On the otherhand it is easy to see that our construction for maximal hypergraphs containing no tight path does indeed contain Berge paths satisfying intersection conditions $(k-2)$. We give the following theorem which is clearly best possible up to a factor of $r$.

Theorem 4.2. Let $\mathcal{H}$ be an $r$-uniform hypergraph containg no Berge path of length $k$ which satisfies intersection conditions $(J)$ in Definition 1.13. If $k-J>r-1$, set $a:=r-1$. Otherwise set $a:=k-J$. Then $e(\mathcal{H})<$ $(k-a)\left(\begin{array}{c}n \\ r-1\end{array}\right)$.

Note that for $J=1$ (i.e. for $(r-1)$-tight paths), the theorem is a weaker result than Theorem 1.7.

Proof of Theorem 4.2. This proof is a simple application of Lemma 4.1.

\section{Acknowledgments}

The first author is partially supported by the Hungarian National Research Fund (grant number NK78439). The second author is partially supported by the Hungarian National Research Fund (grant number K108947). The third author was partially supported by the Department of Energy at Los Alamos National Laboratory under contract DE-AC52-06NA25396, and the DOE Office of Science Advanced Computing Research (ASCR) program in Applied Mathematics.

\section{References}

[1] Allen, P.; Böttcher, J.; Cooley, O.; Mycroft R.: Tight cycles and regular slices in dense hypergraphs, manuscript

[2] Dorbec, P.; Gravier, S.; Sárközy, G.: Monochromatic Hamiltonian ttight Berge-cycles in hypergraphs. Journal of Graph Theory 59, n. 1 (2008) 34-44.

[3] Erdős, P.; and Gallai, T.: On maximal paths and circuits of graphs. Acta Math. Acad. Sci. Hungar. 10, (1959) 337-356. 
[4] Frankl, P.; Füredi, Z.: Exact solution of some Turn-type problems. J. Combin. Theory Ser. A 45 (1987), no. 2, 226262.

[5] Frankl, P.; Katona, G. Y.: Extremal k-edge Hamiltonian hypergraphs. Discrete Math. 308 n. 8 (2008) 1415-1424.

[6] Füredi Z.: Linear paths and trees in uniform hypergraphs Electronic Notes in Discrete Mathematics, 38 (2011) 377-382.

[7] D. Gerbner, C. Palmer: Extremal results for Berge-hypergraphs, arXiv:1505.08127

[8] Glebov, R.; Person Y.; Weps W.: On extremal hypergraphs for Hamiltonian cycles European Journal of Combinatorics, 33:(4), (2012) 544-555.

[9] Győri E.; Katona G. Y. ; Lemons, N.: Hypergraph extensions of the Erdős-Gallai Theorem Electronic Notes in Discrete Mathematics 36 (2006) 655-662.

[10] Katona G. Y.; Kierstead, H. A.: Hamiltonian chains in hypergraphs. J. Graph Theory 30 (1999), no. 3, 205212.

[11] Kühn, D.; Mycroft, R.; Osthus, D.: Hamilton l-cycles in uniform hypergraphs. J. Combin. Theory Ser. A 117 (2010), no. 7, 910927.

[12] Mubayi, D.; Verstraëte, J.: Minimal paths and cycles in set systems. Eurepean Journal of Combinatorics 28 (2007) 1681-1693.

[13] Patkós B.: A note on traces of set families Moscow Journal of Combinatorics and Number Theory, 2:(1) (2012) 47-55.

[14] Rödl, V.: On a packing and covering problem. European J. of Combinatorics 6, (1985) 69-78.

[15] Tuza Zs.: Steiner systems and large non-hamiltonian hypergraphs Matematiche (Catania), 61 (2006), 173180. 